Research Article

\title{
Impact of Copper Oxide and Selenium Nanoparticles on the Activities of Myeloperoxidase and Gamma- Glutamyl Transferase Related Oxidative Stress of Myocardial Infarction Patients
}

\author{
Nada Ahmed ${ }^{1}$, Dhia Hussain ${ }^{1}$, Sura Abdulsattar ${ }^{2}$ \\ ${ }^{1}$ Department of Chemistry, College of Science, Mustansiriyah University, Iraq. \\ ${ }^{2}$ Department of Chemistry and Biochemistry, College of Medicine, Mustansiriyah University, Iraq. \\ —Corresponding author. E-mail: sura742003@yahoo.com; sura742003a@gmail.com
}

Received: Jul. 25, 2020; Accepted: Mar. 22, 2021; Published: May 6, 2021

Citation: Nada Ahmed, Dhia Hussain, and Sura Abdulsattar, Impact of Copper Oxide and Selenium Nanoparticles on the Activities of Myeloperoxidase and Gamma-Glutamyl Transferase Related Oxidative Stress of Myocardial Infarction Patients. Nano Biomed. Eng., 202I, 13(2): 165I7I.

DOI: $10.5101 /$ nbe.v13i2.p165-171.

\begin{abstract}
Nanomedicine has tremendous prospects for the improvement of the diagnosis and treatment of human diseases. Nanotechnology has the potential to revolutionize a wide array of tools in biotechnology so that they are more personalized, portable, cheaper, safer and easier to administer. The present study aims to study the impact of copper oxide and selenium nanoparticles on the oxidative stress in sera of myocardial infraction patients through the synthesis and characterization of copper oxide and selenium nanoparticles, and the evaluation of the status of oxidative stress in sera of myocardial infraction patients through measurement of the activities of myeloperoxidase and gammaglutamyl transferase in the presence and absence of nanoparticles in sera of myocardial infraction patients. Cupric oxide nanoparticles and selenium nanoparticles have been prepared by a chemical method and by laser ablation method respectively, and have been characterized. The result indicated that copper oxide (grain shapes with the average size 30 - $80 \mathrm{~nm}$ ) and selenium nanoparticles (rodshaped structures with the average size $70-90 \mathrm{~nm}$ ) had an inhibition effects on myeloperoxidase and gamma-glutamyl transferase activities, and as a result had an inhibition effects on oxidative stress.
\end{abstract}

Keywords: Copper oxide nanoparticles, Selenium nanoparticles, Myeloperoxidase, Gamma-glutamyl transferase, Myocardial infarction

\section{Introduction}

Nanotechnology deals with production, manipulation and use of material ranging in nanometers [1]. Nanoparticles (NPs) have unusual physical, chemical and optoelectronic properties. The properties of nanoparticles are determined by their huge surfaceto-volume ratio, great surface energy and geometrical constraints [2]. Nanoparticles have wide range of applications in areas such as medical treatments, mechanics, industries, electronics, optics, space industries, drug-gene delivery and energy science [3, 4]. Copper $(\mathrm{Cu})$ is an essential metal for living systems and is found in an assortment of enzymes, including superoxide dismutase (SOD), ferroxidases, and cytochrome oxidase [5]. Copper oxide nanoparticles (CuO NPs) possess a wide range of applications. Compared with ordinary copper oxide powder, the nanoparticles of copper oxide show superior catalytic activity and selectivity [6]. The CuO NPs are used 
in removal of dyes [7], solar energy transformation, heat transfer, and many more [8]. Copper oxide nanoparticles may be toxic for mammalian cells as well as for vertebrates and invertebrates. The main toxicity process relies on $\mathrm{Cu}$ participates in Fenton and Haber-Weiss reactions, facilitating the formation of reactive oxygen species (ROS) and oxidative stress [9]. To protect biological systems from free radical toxicity, several cellular antioxidant defense mechanisms exist to regulate the production of ROS, including enzymatic and nonenzymatic pathways [10]. Several studies reported on therapeutic activities of CuO NPs such as anticancer [11], antibacterial [12], and antioxidant [13]. Selenium is an essential trace element and has been shown to be a natural component in the enzymes glutathione peroxidases and other proteins [5]. In the beginning, the scientific community thought that selenium was toxic, but in 1950s it was found that selenium is an essential element for living organisms, meaning that organisms cannot produce it, so it is necessary to be obtained from the diet [14]. For several reasons, researchers chose Se because of its application in various industries such as medicinal, chemical, electrical and electronics, ceramic, metallurgical, and glass making [15]. Selenium is one of the essential trace elements in the body in due to its anti-oxidative as well as pro-oxidative effect and has great importance in nourishment and medicine $[16,17]$. Selenium exerts a chemo preventive effect at supranutritional doses through multiple mechanisms, such as induction of glutathione S-transferase (GST) to detoxify electrophilic compounds formed during xenobiotic metabolism $[18,19]$ and accumulation of selenocompounds of small molecular weight capable of exerting cytotoxic effects [20]. Although highly effective in chemoprevention, the supranutritional doses of Se which are 10-fold more than the normal physiological requirement are in fact approaching the toxic level of Se [21]. Myeloperoxidase (MPO) is a white blood cell enzyme that has been demonstrated to have complex mechanistic relations with vulnerable plaque development. Myeloperoxidase (MPO) is an inflammatory marker, elevated in acute coronary syndromes (ACSs), especially in acute myocardial infarction (AMI) cases through it acts as a master enzyme in the generation of a range of ROS by catalyzing the conversion of hydrogen peroxide $\left(\mathrm{H}_{2} \mathrm{O}_{2}\right)$ to species including $\mathrm{OH}$, ONOO-, hypochlorous acid $(\mathrm{HOCl})$, and $\mathrm{NO}_{2}[22,23]$. Gamma-glutamyl transferase (GGT) is an enzyme located on the external surface of cellular membranes. Increased GGT activity is a marker of antioxidant inadequacy and increased oxidative stress [24]. Cleavage of glutathione the main thiol antioxidant in humans is the most important physiological function of GGT [25, 26]. There are few studies that have examined the effect of nanoparticles on the oxidative stress. Hence the present study aims to study the effect of copper oxide and selenium nanoparticles on activates of GGT and myeloperoxidase as related oxidative stress in sera of patients with myocardial infarction (MI).

\section{Experimental}

\section{Preparation of copper oxide (CuO) nanoparticles by chemical reaction}

Re-distilled water was used throughout the experiment. In a typical procedure, $1.5 \mathrm{~g}$ of $\mathrm{Cu}_{2}$ $\left(\mathrm{CH}_{3} \mathrm{COOH}\right)_{4}$ was dissolved in $50 \mathrm{~mL}$ of PVC $1 \mathrm{wt} \%$. The solution was added into a round-bottom flask with stirring. The color of the mixture was blue. About 15 $\mathrm{mL}$ of $\mathrm{NaOH}(1 \mathrm{M})$ was rapidly added to the mixture, and a Nano powder suspension was formed. The suspension was kept at $75{ }^{\circ} \mathrm{C}$ for $1 \mathrm{~h}$. A large amount of black precipitate was produced. After cooling to room temperature, the particles were separated by centrifugation and were washed with distilled water to remove any contaminations. The particles were then dried in an oven at $80^{\circ} \mathrm{C}$ [7].

\section{Preparation of selenium (Se) nanoparticles}

Selenium nanoparticles were produced by laser ablation using Se pressed pellet with a diameter of $1 \mathrm{~cm}^{2}$ in distilled water at room temperature. The Se target was placed at the bottom of glass vessel filled with $5 \mathrm{~mL}$ of solution above the target. The colloidal solutions are synthesized by irradiating of Se pellet with pulsed Nd: YAG laser (type HUAFEI) operated of a wavelength $532 \mathrm{~nm}, 7 \mathrm{~ns}$ pulse width and $1 \mathrm{~Hz}$ repetition. The laser energy used for ablation 500 $\mathrm{mJ} /$ pulse). The laser beam was focused at the target surface by using the converging lens of $10 \mathrm{~cm}$ focal length. The diameter of the laser beam on the pellet 2.3 $\mathrm{mm}$. The solution was changed from colorless to color with the increase of the number of laser pulses [27].

\section{Characterization of nanoparticles}

Copper oxide nanoparticles and Se nanoparticles (NPs) have been characterized through measurements of tunneling microscopy, X-ray direction (XRD), atomic force micros- copy (AFE) and field emission scanning electron microscope (FESEM). The crystallite 
size was calculated by using the Debye-Scherer's relation [3].

\section{Subjects}

The study consisted of in total 105 subjects divided into two groups (30 control and 75 patient), with myocardial attending Ibn El-Nafees Hospital, Baghdad Teaching Hospital/Medical. A venous blood sample (10 mL) was collected at morning, then immediately transferred into gel tube and allowed for $10 \mathrm{~min}$ to coagulate at room temperature then centrifuge at 3000 rpm for $5 \mathrm{~min}$. The resulting serum was separated and stored at $-20^{\circ} \mathrm{C}$ until an assay.

\section{Determination of myeloperoxidase activity by ELISA kit}

Myeloperoxidase was measured by using a sandwich enzyme-linked immunosorbent assay (ELISA) kit that measures MPO in serum samples. Concentrations of standard solution of MPO kit were 0, 1.875, 3.75, .5, 15, 30, 60 and $120 \mathrm{ng} / \mathrm{mL}$. The method involved adding $100 \mu \mathrm{L}$ of standards, $100 \mu \mathrm{L}$ of serum samples $+25 \mu \mathrm{L}$ of deuterium-depleted water (DDW) into wells to determine MPO either without $\mathrm{CuO}$ or Se NPs or with the presence of $\mathrm{CuO}$ or Se NPs by adding $100 \mu \mathrm{L}$ of serum samples to $25 \mu \mathrm{L}$ of NPs. The micro titer was plated and incubated at room temperature for $60 \mathrm{~min}$. The plate was kept covered in the dark and level during incubation. Following incubation, the contents of the wells was aspirated. A $100 \mu \mathrm{L}$ of appropriately diluted enzyme antibody conjugate to each well was pipetted, and the plate was kept covered in the dark and level during incubation. A100 $\mu \mathrm{L}$ of TMB substrate solution was pipetted into each well, and incubated in the dark at room temperature for precisely $10 \mathrm{~min}$. After 10 min, $100 \mu \mathrm{L}$ of stop solution was added to each well and the absorbance (450 nm) of the contents of each well was determined.

\section{Determination of gamma-glutamyl transferase activity}

Kinetic colorimetric method for the determination of gamma-glutamyl transferase activity was used. An $80 \mu \mathrm{L}$ of samples $+250 \mu \mathrm{L}$ of DDW was placed into the tube to determine GGT either without $\mathrm{CuO}$ or Se NPs or with the presence of $\mathrm{CuO}$ or Se NPs by adding $80 \mu \mathrm{L}$ of serum samples to $20 \mu \mathrm{L}$ of NPs. Then, $1 \mathrm{~mL}$ of buffer was added to each tube with mixing and incubated foe $1 \mathrm{~min}$ at $37^{\circ} \mathrm{C}$. A $250 \mu \mathrm{L}$ of L-gamma glytamyl-3-carboxy-4-nitronilide was used as substrate, mixed; then, the absorbance was read after
$1 \mathrm{~min}$, and at the same time the stopwatch was started; the absorbance was read again exactly after 1, 2 and 3 $\min$.

\section{Statistical analysis}

SPSS version 23 Statistical Microsoft Office was used for analyzing the research data. Student t-test was used to evaluate the differences in the mean values, where $\mathrm{p}<0.05$ indicated a significant value.

\section{Results and Discussion}

XRD patterns of the $\mathrm{CuO}$ film nanostructures obtained is shown in Fig. 1. All the peaks can be indexed to the polycrystalline phase of $\mathrm{CuO}$ (JCPDS 48-1548) and no peaks of impurities were detected, indicating that the $\mathrm{CuO}$ nanostructures were pure and well crystallized. The XRD patterns of $\mathrm{CuO}$ contained three main peaks at diffraction angles of $35.2^{\circ}, 38.4^{\circ}$ and $48.7^{\circ}$, corresponding to (111), (200) and (220), respectively. The crystallite size was calculated, and the results were shown in Table 1 . The intensities and positions of peaks are in good agreement with the values reported by Lanje et al. [28].



Fig. 1 XRD patterns of the $\mathrm{CuO}$ nanoparticles.

FE-SEM micrographs of the sample CuO NPs synthesized chemical reduction method was demonstrated in Fig. 2, which shows the microscopic structure and morphology of the $\mathrm{CuO}$ nanoparticles are in agreement with the XRD results. This image reveals that the resulting nanocrystals are well-dispersed, uniform, with narrow particle size distribution, and clearly defined grain shapes and diameters of most of them are in the range of $30-80 \mathrm{~nm}$.

The three-dimension AFM images are shown in Fig. 3. The topography of the surfaces of the prepared membranes employed AFM, the imaging and analysis capacity of these surfaces, and the statistical accuracy 
Table 1 Summery of X-ray characterization of CuO NPs ablation in DDW with different laser pulses

\begin{tabular}{ccccccc}
\hline 2Theta $\left({ }^{\circ}\right)$ & Beta & Cos theta $(\mathrm{rad})$ & Beta (rad) & D (nm) & Dis & Strain \\
\hline 35.5465 & 0.8317 & 0.951779424 & 0.014591 & 9.980104 & 100.3991 \\
38.733 & 0.8233 & 0.942833623 & 0.014444 & 10.17759 & 96.54064 \\
48.8288 & 0.8 & 0.909663644 & 0.014035 & 10.85594 & 84.85266 \\
\hline
\end{tabular}
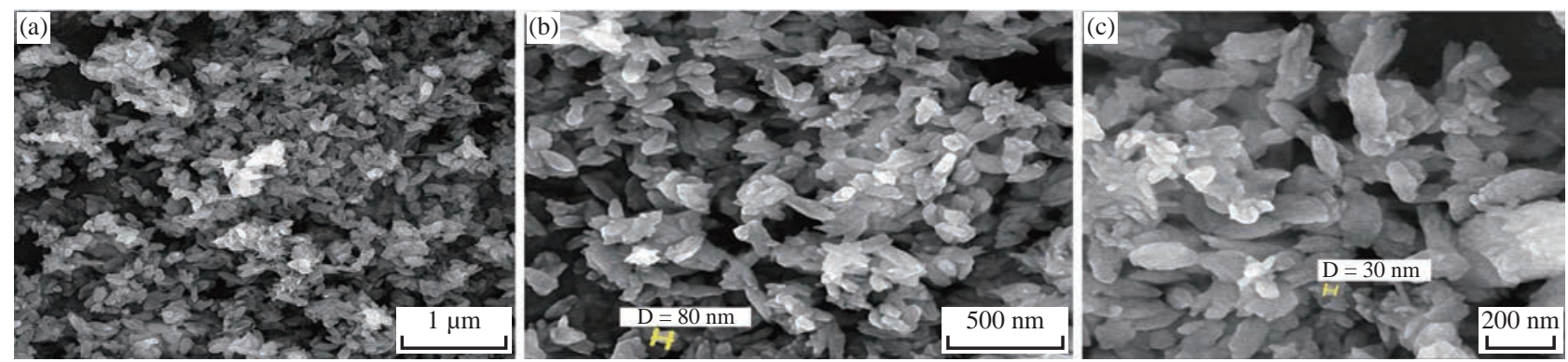

Fig. 2 FE-SEM images of CuO NPs: (a) $30 \mathrm{k} \times$ (b) $60 \mathrm{k} \times$, and (c) $100 \mathrm{k} \times$.

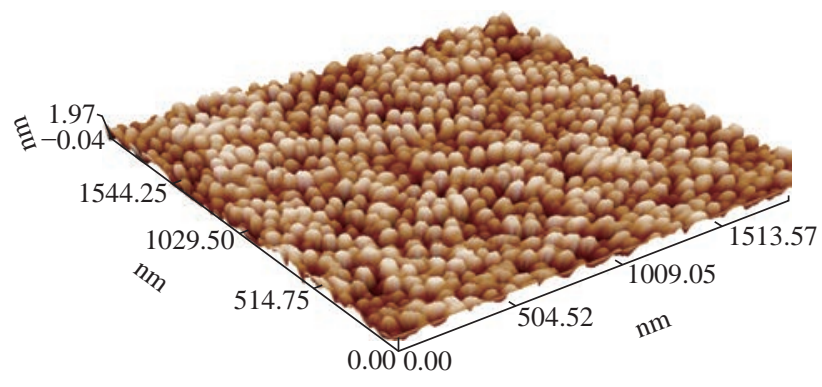

Fig. 3 Three-dimension AFM images.

of roughness values based on RMS.

The XRD diffraction patterns of synthesized Se nanoparticles films ablated in DDW and deposited on glass are shown in Fig. 4. The XRD patterns of Se contain three main peaks at diffraction angles of $23.2^{\circ}$, $29.5^{\circ}$ and $43.2^{\circ}$ corresponded to (100), (101) and (110) respectively.

Field emission scanning electron microscope is one of the best to study the surface quality cross section of the nanostructure. The FE-SEM micrographs result

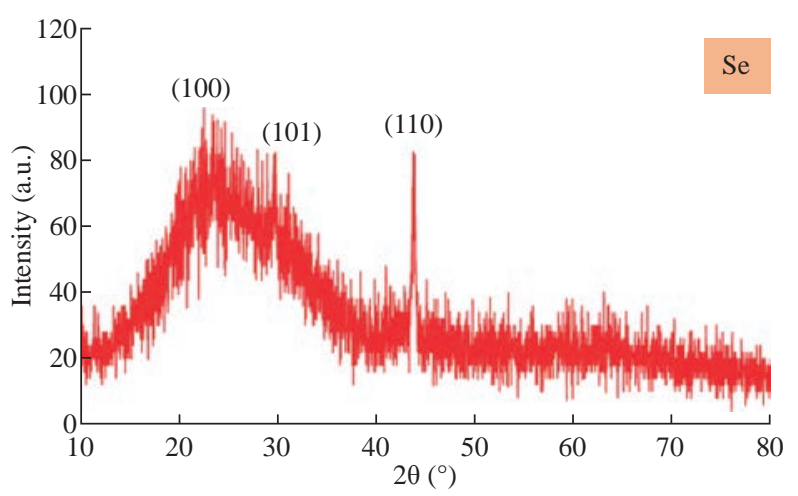

Fig. 4 XRD patterns of the Se nanostructures formed by 100 pulses. demonstrated a smooth homogenous surface as shown in top view image and cross section as in Fig. 5. The FE-SEM image showed a needle shaped nanoparticles with no other types of nanoparticles, with the average size $70-90 \mathrm{~nm}$, which means that the sample was very pure.

Fig. 6 shows the three dimensions of AFM images of Se NPs, by laser ablation of metal immersed in DDW at 100, pulses for Se NPs. Topological analysis was carried out using AFM, which produced topological images of surfaces at a very high magnification and facilitated the observation of the atomic structure of crystals.

\section{Effect of CuO and Se nanoparticles on gamma-glutamyl transferase activity}

The results presented in Fig. 7 show that the nanoparticles had an inhibition effect on the gammaglutamyl transferase activity in the sera of myocardial infarction patients where CuO NPs (48\%) presented more inhibition effect on GGT activity than Se NPs (33\%). The inhibition percentages were calculated by comparing the GGT concentration with and without CuO NPs or SeNPs, as in Equation (1) [29].

$\%$ Inhibition $=100-100 \times($ GGT with NPs $/$ GGT without NPs). (1)

Several studies revealed the effects of copper oxide nanoparticles on tissue accumulation and antioxidant enzymes of Galleria Mellonella L. [30] and Oreochromis niloticus [31]. The previous study of Kojouri et al. indicated the beneficial effects of Se NPs on antioxidant activity and weight gain patterns of newborn lambs which may promote lambs' growth rate 

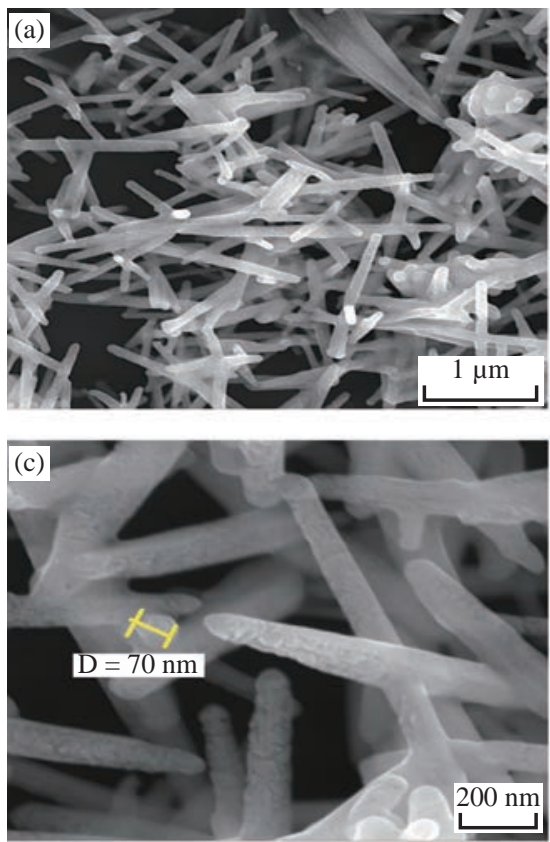
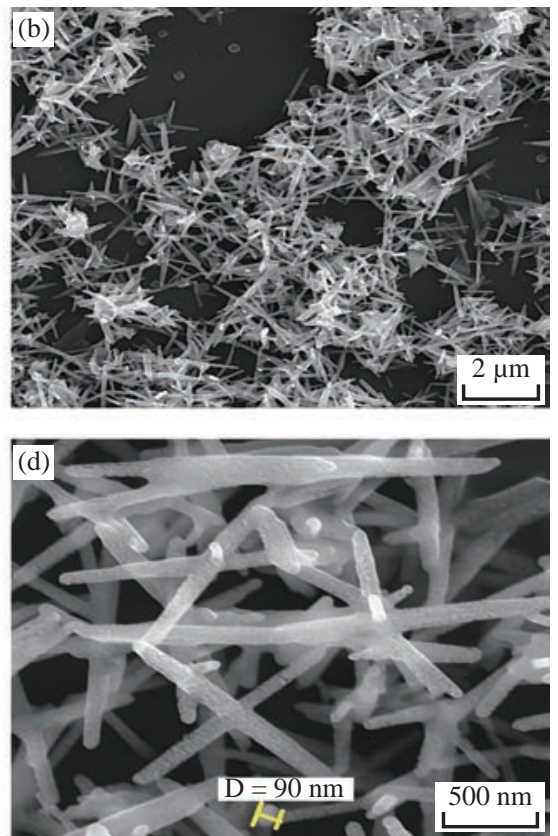

Fig. 5 Field emission scanning electron microscopy of Se nanostructures: (a) $10 \mathrm{k} \times$, (b) $30 \mathrm{k} \times$, (c) $50 \mathrm{k} \times$, and (d) $100 \mathrm{k} \times$.

Table 2 Summery of X-ray characterization for Se NPs ablation in D.D.W with different laser pulses

\begin{tabular}{ccccccc}
\hline 2Theta $\left(^{\circ}\right)$ & Beta & Cos theta $(\mathrm{rad})$ & Beta $(\mathrm{rad})$ & D $(\mathrm{nm})$ & Dis & Strain \\
\hline 23.4841 & 0.7 & 0.978856748 & 0.012281 & 11.52978 & 75.22431 & 3.005262 \\
29.6388 & 0.45 & 0.966392684 & 0.007895 & 18.16653 & 30.30094 & 1.907354 \\
43.7814 & 0.48 & 0.927155782 & 0.008421 & 17.75187 & 31.73304 & 1.951907 \\
\hline
\end{tabular}

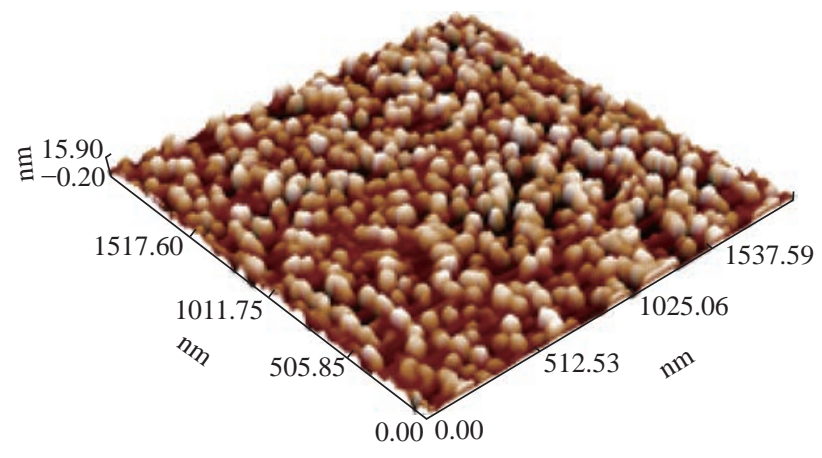

Fig. 6 Three-dimension AFM images of Se NPs.

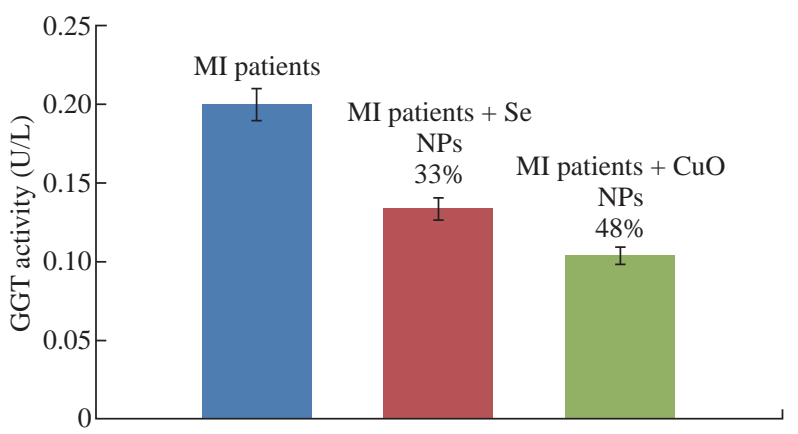

Fig. 7 GGT levels in the sera of MI patients with inhibition percentage in the presence of Se NPs and in the presence of CuO NPs. in the postnatal period [32]. Yuan et al. suggested that SeNPs could be used as a promising anticonvulsant drug due to its potent antioxidant, anti-inflammatory, and neuromodulatory activities [33]. In 2020, Vahdati et al. pointed out the ability of selenium nanoparticles to exhibit anticancer, antioxidant, antibacterial and antibiofilm properties. So far, remarkable antimicrobial activity of these nanoparticles have been evidenced against pathogenic bacteria, fungi and yeasts [34].

According to our knowledge, the present work is the first study that has carried out the effect of copper oxide and selenium nanoparticles on the GGT activity in sera of MI patients. This result was in agreement with the study by Shalby et al. who reported that the enzyme activities of AST, ALT, ALP and GGT in rats revealed significant reduction as a result of the treatment with Se NPs $(-27.74 \%,-43.13 \%,-31.96 \%$ and $-30.18 \%$, respectively) [35]. Moreover our results are in line with previous studies that showed selenium was able to protect hepatic cells from oxidative damage via its free radical scavenging activity [36] plus its ability to enhance the antioxidant protective system [37]. 


\section{Effect of CuO and Se nanoparticles on myeloperoxidase}

The objective of this work is to study the effects of the presence of these NPs on the levels of MPO in pool sera of patients with MI. The study characterized the binding between the anti-MPO antibodies with its antigen in sera patients in the presence affixed size concentration of NPs. It is found that CuO NPs and Se NPs have an inhibitor effect on the binding between anti-MPO antibody and antigen MPO in pool sera of MI patients. Fig. 8.

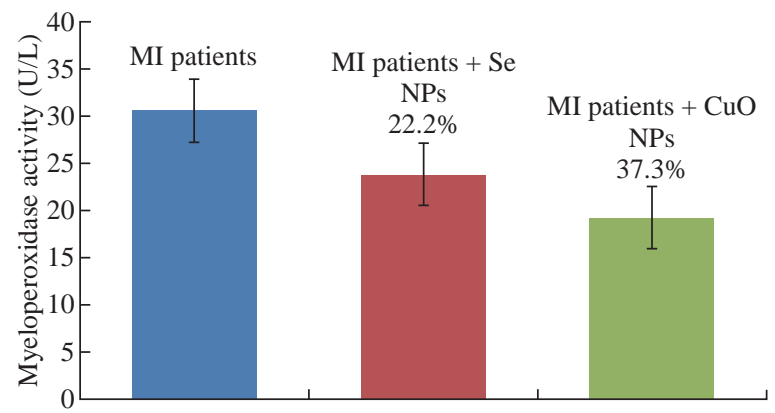

Fig. $8 \mathrm{MPO}$ levels in the sera of MI patients, with inhibition percentage in the presence of Se NPs and in the presence of CuO NPs.

The previous study of Sanfins et al. revealed that in high concentrations of nanoparticles, myeloperoxidase activity is decreased whereas in low concentrations of nanoparticles the activity is increased. The effect of the nanoparticles on myeloperoxidase is dependent on the total protein concentration as low concentrations of bovine serum albumin together with nanoparticles further increase the myeloperoxidase activity [38].

\section{Conclusions}

Copper oxide nanoparticles were synthesized by chemical reduction method as grain shapes with 30$80 \mathrm{~nm}$ in size. Selenium nanoparticles were prepared by laser ablation in needle shaped nanoparticles with the average size of 70 - $90 \mathrm{~nm}$. Both copper oxide and selenium nanoparticles have an inhibition effect on myeloperoxidase and GGT activities which as a result have an inhibition effect on oxidative stress. So, these nanoparticles may play an important role as a drug delivery for treatment of myocardial infraction. Copper oxide nanoparticles are more effective inhibitors than Se NPs on the activity of GGT and myeloperoxidase.

\section{Conflict of Interests}

The authors declare that no competing interest exists.

\section{References}

[1] P. Heera, S. Shanmugam, Nanoparticle characterization and application: an overview. Int. J. Curr. Microbiol. App. Sci, 2015, 4(8): 379-386.

[2] S. Chaudhary, S.K. Mehta, Selenium nanomaterials: applications in electronics, catalysis and sensors. $J$. Nanosci. Nanotechnol., 2014, 14(2): 1658-1674.

[3] S. Iravani, H. Korbekandi, S.V. Mirmohammadi, et al., Synthesis of silver nanoparticles: Chemical, physical and biological methods. Res. Pharm. Sci., 2014, 9(6): 385.

[4] S.A. Abdulsattar, A.H. Drywall, Effect of gold and nickel nanoparticles on immune proteins of toxoplasmosis patients. Asian J Pharm Clin Res, 2017, 10(10): 162-164.

[5] A. Yakoh, C. Pinyorospathum, W. Siangproh, et al., Biomedical probes based on inorganic nanoparticles for electrochemical and optical spectroscopy applications. Sensors, 2015, 15(9): 21427-21477.

[6] G.F. Nordberg, B.A. Fowler, and M. Nordberg, Handbook on the toxic. Handbook on the toxicology of metals. Academic press, 2014.

[7] A.M.A. Majeed, A.N. Abd, A.A. Hussein, et al., Fabrication and characterization of copper oxide nanoparticles/PSi heterodiode. Int. Lett. Chem. Phys. Astron., 2015, 57: 25.

[8] M. Ahamed, H.A. Alhadlaq, M.A. Khan, et al., Synthesis, characterization, and antimicrobial activity of copper oxide nanoparticles. J. Nanomater., 2014: 17.

[9] F. Perreault, S.P. Melegari, C.H. da Costa, et al., Genotoxic effects of copper oxide nanoparticles in Neuro 2A cell cultures, Sci. Total Environ., 2012, 441: 117-124.

[10] P. Patlevič, J. Vašková, P. Švorc, et al., Reactive oxygen species and antioxidant defense in human gastrointestinal diseases. Integrative Medicine Research, 2016, 5(4): 250258.

[11] K. Subashini, S. Prakash, and V. Sujatha, Anticancer activity of copper oxide nanoparticles synthesized from brassia actinophylla flower extract. Asian Journal of Chemistry, 2019, 31: 1899-1904.

[12] M.B. Zaman, R. Poolla, P. Singh, et al., Biogenic synthesis of $\mathrm{CuO}$ Nanoparticles using Tamarindus indica L. and a study of their photocatalytic and antibacterial activity. Environmental Nanotechnology, Monitoring \& Management, 2020: 100346.

[13] D. Rehana, D. Dharmasivam, S. Kumar, et al., Evaluation of antioxidant and anticancer activity of copper oxide nanoparticles synthesized using medicinally important plant extracts. Biomedicine \& Pharmacotherapy, 2017, 89: 1067-1077.

[14] Y.W. Baek, Y.J. An, Microbial toxicity of metal oxide nanoparticles (CuO, $\mathrm{NiO}, \mathrm{ZnO}$, and $\mathrm{Sb}_{2} \mathrm{O}_{3}$ ) to Escherichia coli, Bacillus subtilis, and Streptococcus aureus. Sci. Total Environ., 2011, 409(8): 1603-1608.

[15] R. Sankar, R. Maheswari, S. Karthik, et al., Anticancer activity of Ficus religiosa engineered copper oxide nanoparticles. Mater. Sci. Eng. C, 2014, 44: 234-239.

[16] S. Chaudhary, A. Umar, and S.K. Mehta, Selenium nanomaterials: an overview of recent developments in synthesis, properties and potential applications. Prog. Mater. Sci., 2016, 83: 270-329.

[17] K. Donaldson, V. Stone, Current hypotheses on the mechanisms of toxicity of ultrafine particles. Ann. Ist. Super. Sanita, 2003, 39(3): 405-410.

[18] K. Bayoumy, R. Sinha, Molecular chemoprevention by selenium: A genomic approach. Mutation Research, 2006, 591: 224-236.

[19] W.M. El-Sayed, T. Aboul-Fadl, J.G. Lamb, et al., Effect of selenium-containing compounds on hepatic chemoprotective enzymes in mice. Toxicology, 2006, 
220: 179-188.

[20] P.D. Whanger, Selenium and its relationship to cancer: An update. Br J Nutr., 2004, 91(1): 11-28.

[21] M.F. Raisbeck, Food animal pract. Vet. Clin. North Am., 2000, 16: 465-480.

[22] V.M. Vinodhini,, K.B. Sudhan, Gamma-glutamyl transferase as an indicator of obesity: A cross-sectional study. Asian J Pharm Clin Res, 2016, 9(Suppl. 3): 240242.

[23] H.S. Abdulghani, S.A. Abdulsattar, and E.N. Salman, Association of serum myeloperoxidase level with risk of coronary artery disease in patients with type 2 diabetes. Indian Journal of Forensic Medicine \& Toxicology, 2020, 4(3): 384-391.

[24] N.N. Ahmed, D.H. Hussain, and S.A. Abdulsattar, Myeloperoxidase and gamma-glutamyl transferase as oxidative stress marker sera of myocardial infarction patient. Biochem. Cell. Arch., 2019, 19(Supplement 1): 2655-2659.

[25] G. Ndrepepa, R. Colleran, and A. Kastrati, Gammaglutamyl transferase and the risk of atherosclerosis and coronary heart disease. Clin. Chim. Acta, 2017.

[26] S. Sugiyama, Y. Okada, G.K. Sukhova, et al., Macrophage myeloperoxidase regulation by granulocyte macrophage colony-stimulating factor in human atherosclerosis and implications in acute coronary syndromes. Am. J. Pathol., 2001, 158(3): 879-891.

[27] S. Chaudhary, A. Umar, and S.K. Mehta, Selenium nanomaterials: an overview of recent developments in synthesis, properties and potential applications. Prog. Mater. Sci., 2016, 83: 270-329.

[28] A.S. Lanje, S.J. Sharma, R.P. Pode, et al., Synthesis and optical characterization of copper oxide nanoparticlesAdv. Appl. Sci. Res., 2010, 1(2): 36-40.

[29] A. Shapiro, Re: How to find the percentage inhibition for each concentration of deprenyl tested?. Retrieved from <https://www.researchgate.net/post/How_to_find_ the_percentage_inhibition_for_each_concentration_of_ deprenyl_tested/565665e4614325eeb18b4577/citation/ download>, 2015.

[30] B.S. Tuncsoy, M. Tuncsoy, T. Gomes, et al., Effects of copper oxide nanoparticles on tissue accumulation and antioxidant enzymes of Galleria mellonella L.. Bull Environ Contam Toxicol, 2019, 102: 341-346.

[31] M. Tunçsoy, S. Duran, Ö Ay, et al., Effects of copper oxide nanoparticles on antioxidant enzyme activities and on tissue accumulation of oreochromis niloticus. Bull Environ Contam Toxicol, 2017, 99(3): 360-364.

[32] G. Kojouri, F. Arbabi, and A. Mohebbi, The effects of selenium nanoparticles (SeNPs) on oxidant and antioxidant activities and neonatal lamb weight gain pattern. Comp Clin Pathol, 2020, 29: 3693374.

[33] X. Yuan, Z. Fu, P. Ji, et al., Selenium nanoparticles pretreatment reverse behavioral, oxidative damage, neuronal loss and neurochemical alterations in pentylenetetrazoleinduced epileptic seizures in mice. Int J Nanomedicine, 2020, 15: 6339-6353.

[34] M. Vahdati, T. Moghadam, Synthesis and characterization of selenium nanoparticles-lysozyme nanohybrid system with synergistic antibacterial properties. Sci Rep, 2020, 10: 510 .

[35] A.B. Shalby, M.D. Abd El-Maksoud, A.E. Abdel Moneim, et al., Antifibrotic candidates of Selenium nanoparticles and selenium in the experimental model. J App Pharm Sci., 2017, 7(9): 191-198.

[36] N.M. Shafik M.M. El Batsh, Protective effects of combined selenium and Punica granatum treatment on some inflammatory and oxidative stress markers in arsenic-induced hepatotoxicity in rats. Biol Trace Elem Res, 2016, 169: 121-128.

[37] A.A. Newairy, A.S. El-Sharaky, M.M. Badreldeen, et al., The hepatoprotective effects of selenium against cadmium toxicity in rats. Toxicology, 2007, 242: 23-30.

[38] E. Sanfins, A. Correia, S.B. Gunnarsson, et al., Nanoparticle effect on neutrophil produced myeloperoxidase. PLOS ONE, 2018, 13(1).

Copyright $(\subset$ Nada Ahmed, Dhia Hussain, and Sura Abdulsattar. This is an open-access article distributed under the terms of the Creative Commons Attribution License, which permits unrestricted use, distribution, and reproduction in any medium, provided the original author and source are credited. 